\title{
Approximation of Analytic Functions with Prescribed Boundary Conditions by Circle-Packing Maps*
}

\author{
T. Dubejko ${ }^{\dagger}$ \\ Department of Mathematics, University of Tennessee, \\ Knoxville, TN 37996-1300, USA \\ and \\ Mathematical Sciences Research Institute, \\ Berkeley, CA 94720, USA
}

\begin{abstract}
We use recent advances in circle-packing theory to develop a constructive method for the approximation of an analytic function $F: \Omega \rightarrow \mathrm{C}$ by circle packing maps providing we have only been given $\Omega,\left.\left|F^{\prime}\right|\right|_{\partial \Omega}$, and the set of critical points of $F$. This extends the earlier results of Carter and Rodin and of Colin de Verdière and Mathéus, for functions $F$ with no critical points.
\end{abstract}

\section{Introduction}

In [CR] and [CVM] an inverse problem for circle packings and locally univalent functions was considered. It was shown that, for a given bounded simply connected domain $\Omega$ and a positive continuous function $\lambda: \partial \Omega \rightarrow(0, \infty)$, one can construct a sequence of locally univalent hexagonal circle packings and, associated with it, a sequence $\left\{f_{n}\right\}$ of circlepacking maps such that $f_{n} \rightarrow f$ uniformly on compacta of $\Omega$ as $n \rightarrow \infty$, where $f$ is the unique (up to some standard normalization) locally univalent analytic function in $\Omega$ with $\left|f^{\prime}\right|: \bar{\Omega} \rightarrow(0, \infty)$ being continuous and $\left.\left|f^{\prime}\right|\right|_{\partial \Omega} \equiv \lambda$.

Here we are interested in a generalization of the above problem, that is in an approximation of an analytic function $F: \Omega \rightarrow \mathrm{C}$ via circle-packing maps, where $F$ has a finite set of critical points in $\Omega$ and $\left|F^{\prime}\right|: \bar{\Omega} \rightarrow[0, \infty)$ is continuous with $\left|F^{\prime}\right| \equiv \lambda$ on $\partial \Omega$. We prove that this can be achieved, roughly speaking, by taking the circle-packing map from

\footnotetext{
* The author gratefully acknowledges support of the Tennessee Science Alliance and the National Science Foundation. Research at MSRI is supported in part by Grant No. DMS-9022140.

${ }^{\dagger}$ Current address: Department of Mathematics, Northwestern University, Evanston, IL 60208, USA. tdubejko@math.nwu.edu.
} 
the portion of a regular hexagonal circle packing of a suitably small mesh that fills up $\Omega$ to a combinatorially equivalent branched circle packing, whose branch set approximates the set of critical points of $F$ and whose boundary circles have their radii determined by $\lambda$ and the mesh. The precise setup is laid out in Section 3 and the main result is contained in Theorem 3.1.

The techniques we employ here to verify our approximation scheme are ideologically quite different from the ones used in [CR] and [CVM]; the latter are closely linked with hexagonal combinatorics and with the local univalence of functions considered, the former are based on advances in the theory of circle packing. In fact, the arguments presented in this paper can be easily extended to patterns other than hexagonal (see [HR] and [St]).

As the proof of Theorem 3.1 relies heavily on results from the theory of circle packing, we combine them, together with an introduction of basic terminology, in Section 2. The reader who would like to obtain more information about the subject should see [BS] or [D1].

The author would like to acknowledge that both figures in this paper have been created with the help of CirclePack, a software package developed by Ken Stephenson.

\section{Circle-Packing Overview}

Let $\mathbb{K}$ be a simplicial 2-complex which is a triangulation of a simply connected domain in the complex plane $\mathbf{C}$ and which has its orientation induced from the orientation of the plane. Write $\mathbb{K}^{(0)}$, int $\mathbb{K}^{(0)}$, bd $\mathbb{K}^{(0)}, \mathbb{K}^{(1)}$, and $\mathbb{K}^{(2)}$ for the sets of vertices, interior vertices, boundary vertices, edges, and faces of $\mathbb{K}$, respectively. A collection $\mathcal{P}$ of circles in $\mathbf{C}$ is said to be a circle packing for $\mathbb{K}$ if there is one-to-one correspondence between the elements of $\mathbb{K}^{(0)}$ and $\mathcal{P}\left(\mathbb{K}^{(0)} \ni v \leftrightarrow C(v) \in \mathcal{P}\right)$ such that if $\langle u, v\rangle \in \mathbb{K}^{(1)}$, then $C(u)$ and $C(v)$ are externally tangent and if $\langle u, v, w\rangle \in \mathbb{K}^{(2)}$ is a positively oriented triple, then so is $\langle C(u), C(v), C(w)\rangle$.

Let $S_{\mathcal{P}}$ be a simplicial map $S_{\mathcal{P}}: \mathbb{K} \rightarrow \mathbf{C}$ which is first defined on $\mathbb{K}^{(0)}$ by mapping $v \in \mathbb{K}^{(0)}$ to the euclidean center of $C(v) \in \mathcal{P}$ and is then extended affinely to $\mathbb{K}^{(1)}$ and $\mathbb{K}^{(2)}$ via barycentric coordinates. If $\Delta \in \mathbb{K}^{(2)}$ and $v$ is a vertex of $\Delta$, then $\alpha_{\mathcal{P}}(v, \Delta)$ will denote the angle at the vertex $S_{\mathcal{P}}(v)$ in the euclidean triangle $S_{\mathcal{P}}(\Delta)$. We define the carrier of $\mathcal{P}$ as $\operatorname{carr}(\mathcal{P}):=S_{\mathcal{P}}(\mathbb{K})$ and the angle sum of $\mathcal{P}$ at a vertex $v$ as $\Theta_{\mathcal{P}}(v):=\sum_{\Delta \in \mathbb{K}^{(2)}} \alpha \mathcal{P}(v, \Delta)$. It follows from our definition of a circle packing that $\Theta_{\mathcal{P}}(v)$ is a positive integer multiple of $2 \pi$ if $v$ is an interior vertex of $\mathbb{K}$. Moreover, if $\mathcal{P}$ is univalent, i.e., the circles of $\mathcal{P}$ have mutually disjoint interiors, then $\Theta_{\mathcal{P}}(v)=2 \pi$ for each $v \in$ int $\mathbb{K}^{(0)}$. However, the converse is not true (see Fig. 1(b)). If $v \in$ int $\mathbb{K}^{(0)}$ and $\Theta_{\mathcal{P}}(v)=2 \pi n, n \geq 2$, then $v$ is called a branch vertex (point) of $\mathcal{P}$ of order $(n-1)$ (multiplicity $n$ ); geometrically this means that the circles in $\mathcal{P}$ associated with the neighboring vertices of $v$ wrap $n$-times around $C(v)$. The listing of all the branch vertices of $\mathcal{P}$ together with their orders is called the branch set of $\mathcal{P}$ and is denoted $\operatorname{br}(\mathcal{P})$. A circle packing without branch points is called locally univalent.

So far we have talked about circle packings but we have not said anything about their existence. Before we can state necessary and sufficient conditions for the existence we need the following definition, in which $\mathbf{N}$ denotes the set of nonnegative integers. 

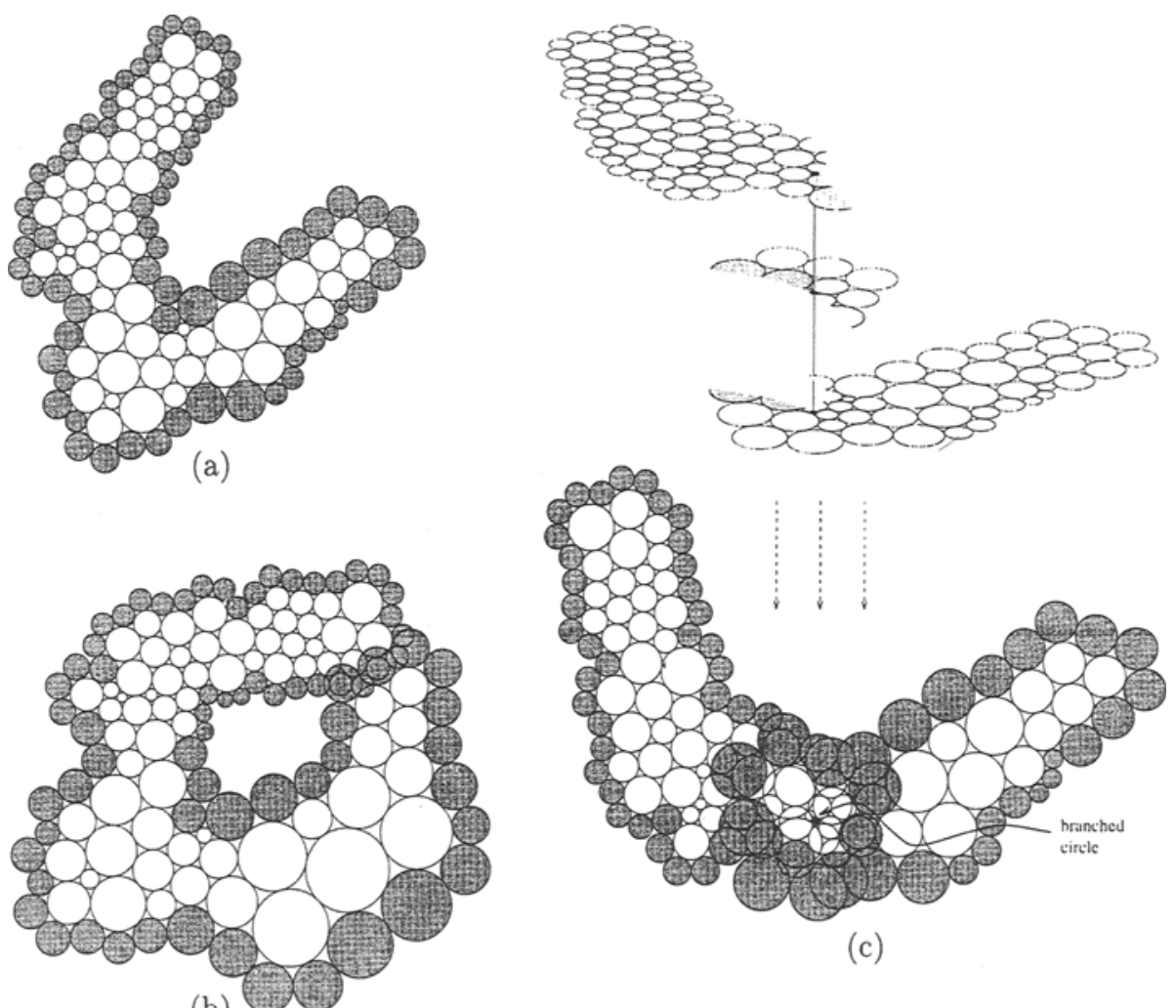

(c)

Fig. 1. Different packings of the same complex: (a) univalent, (b) locally univalent, and (c) branched (and its decomposition into univalent sheets).

Definition 2.1. Let $\mathbb{K}$ be a finite or infinite triangulation of a simply connected domain in $\mathbf{C}$. A set $\left\{\left(v_{1}, l_{1}\right),\left(v_{2}, l_{2}\right), \ldots\right\} \subset$ (int $\left.\mathbb{K}^{(0)}\right) \times \mathbf{N}$, possibly finite, is called a branch structure for $\mathbb{K}$ if every simple closed edge-path $\Gamma$ in $\mathbb{K}$ has at least $2 \ell(\Gamma)+3$ edges, where $\ell(\Gamma)=\sum_{i=1}^{n} \delta_{i}(\Gamma) l_{i}$ and $\delta_{i}(\Gamma)$ is equal to 1 if $v_{i}$ is enclosed by $\Gamma$ and 0 otherwise.

The following theorem answers the existence question for circle packings (see [D1] and [D2]); in the case when the branch set is empty this result is due to Koebe.

Theorem 2.2. Suppose $\mathbb{K}$ is a triangulation of a simply connected domain in $\mathbf{C}$. Let $b_{1}, \ldots, b_{m}$ be interior vertices of $\mathbb{K}$, and let $k_{1}, \ldots, k_{m}$ be nonnegative integers. Then there exists a circle packing for $\mathbb{K}$ with branch set $\mathfrak{B}=\left\{\left(b_{1}, k_{1}\right), \ldots,\left(b_{m}, k_{m}\right)\right\}$ if and only if $\mathfrak{B}$ is a branch structure for $\mathbb{K}$.

Notice that when $0=k_{1}=\cdots=k_{m}$, then $\mathfrak{B}$ is a trivial case of a branch structure; in fact the number of pairs $\left(b_{i}, k_{i}\right)$ in $\mathfrak{B}$ is irrelevant in this case. Therefore we identify the sets $\left\{\left(b_{1}, 0\right),\left(b_{2}, 0\right), \ldots\right\}$ with the empty set. 
For the purposes of this paper we need a stronger version of Theorem 2.2 when $\mathbb{K}$ is finite which will be stated shortly, but first we have to introduce a function which is quite handy when working with circle packings. If $\mathcal{P}$ is a circle packing for $\mathbb{K}$, then a function $r_{\mathcal{P}}: \mathbb{K}^{(0)} \rightarrow(0, \infty)$ defined by $r_{\mathcal{P}}(v):=$ the euclidean radius of $C(v)$ is called the radius function of $\mathcal{P}$. Now we give a stronger version of Theorem 2.2 (see [D1]).

Theorem 2.3. Suppose $\mathbb{K}$ is a finite triangulation of a simply connected domain in C. Let $b_{1}, \ldots, b_{m}$ be interior vertices of $\mathbb{K}$, and let $k_{1}, \ldots, k_{m}$ be nonnegative integers. For any given $\rho:$ bd $\mathbb{K}^{(0)} \rightarrow(0, \infty)$ there exists a circle packing $\mathcal{P}$ for $\mathbb{K}$ with branch set $\mathfrak{B}=\left\{\left(b_{1}, k_{1}\right), \ldots,\left(b_{m}, k_{m}\right)\right\}$ and with $\left.r_{\mathcal{P}}\right|_{\mathrm{bd}} \mathbb{K}^{(0)} \equiv \rho$ if and only if $\mathfrak{B}$ is a branch structure for $\mathbb{K}$. Moreover, $\mathcal{P}$ is unique up to isometries of $\mathbf{C}$.

Suppose that $\mathcal{P}$ and $\mathcal{Q}$ are circle packings for $\mathbb{K}$. Then $\mathcal{P}$ and $\mathcal{Q}$ are called combinatorially equivalent (or, shortly, c-equivalent) with complex $\mathbb{K}$. The map $F_{\mathcal{P}, \mathcal{Q}}:=$ $S_{\mathcal{Q}} \circ S_{\mathcal{P}}{ }^{-1}: \operatorname{carr}(\mathcal{P}) \rightarrow \operatorname{carr}(\mathcal{Q})$ is called the circle-packing map from $\mathcal{P}$ to $\mathcal{Q}$ (shortly, cp-map). The map $F_{\mathcal{P}, \mathcal{Q}}^{\#}: \operatorname{carr}(\mathcal{P}) \rightarrow(0, \infty)$ defined on the set of vertices of $S_{\mathcal{P}}(\mathbb{K})$ by $F_{\mathcal{P}, \mathcal{Q}}^{\#}\left(S_{\mathcal{P}}(v)\right)=r_{\mathcal{Q}}(v) / r_{\mathcal{P}}(v)$ and then extended affinely to faces of $S_{\mathcal{P}}(\mathbb{K})$ is called the ratio function from $\mathcal{P}$ to $\mathcal{Q}$. The ratio function allows the "distortion" of circle packings to be measured or to see how distinct two packings are. This is due to the following rather elementary fact (implied by monotonicity properties of the angle sums-see [D1, Theorem. 2.5 (Proof)] and [G]), which gives, for example, the uniqueness in Theorem 2.3.

\section{Fact 2.4.}

(1) If $\mathcal{P}$ and $\mathcal{Q}$ are circle packings for $\mathbb{K}$ with identical branch sets (possibly empty), then $F_{\mathcal{P}, \mathcal{Q}}^{*}$ does not attain its infimum or supremum in int $\mathbb{K}^{(0)}$ unless it is constant.

(2) If $\mathcal{P}$ and $\mathcal{Q}$ are circle packings for $\mathbb{K}$ such that $\operatorname{br}(\mathcal{P})=\emptyset$ and $b r(\mathcal{Q}) \neq \emptyset$, then $F_{\mathcal{P}, \mathcal{Q}}^{\#}$ does not attain its supremum in int $\mathbb{K}^{(0)}$.

We now recall some results regarding circle packings and approximation of analytic functions. Let $\mathbb{H}$ be the regular hexagonal 2-complex of mesh 2 with vertices at $2 k+$ $l(1+\sqrt{3} i), k, l \in \mathbf{Z}$. Write $\mathbb{H}_{n}:=(1 / n) \mathbb{H}$. Let $\mathcal{P}_{n}$ be the univalent circle packing whose carrier is $\mathbb{H}_{n}$, i.e., the regular hexagonal circle packing of circles of radius $1 / n$ and with vertices at $k / n+l(1+\sqrt{3} i) / n, k, l \in \mathbf{Z}$. We notice that if $\mathfrak{B}=\left\{\left(b_{1}, k_{1}\right), \ldots,\left(b_{m}, k_{m}\right)\right\}$ is a branch structure for $\mathbb{H}_{n}, n \geq 1$, then necessarily $k_{1}=\cdots=k_{m}=1$, i.e., the $b_{i}$ 's are simple branch points. Thus, when dealing with complexes $\mathbb{H}_{n}$, we adopt a convention by writing $\left\{b_{1}, \ldots, b_{m}\right\}$ for $\left\{\left(b_{1}, 1\right), \ldots,\left(b_{m}, 1\right)\right\}$. We now recall the definition of a discrete complex polynomial.

Definition 2.5. A map $f: \mathbf{C} \rightarrow \mathbf{C}$ is a discrete complex polynomial for $\mathbb{H}_{n}, n \geq 1$, with the branch set $\mathfrak{B}=\left\{b_{1}, \ldots, b_{m}\right\}$ if the following are satisfied:

(i) There exists a circle packing $\mathcal{B}$ for $\mathbb{H}_{n}$ with the branch set $\mathfrak{B}$ such that $f$ is the cp-map from $\mathcal{P}_{n}$ to $\mathcal{B}$.

(ii) $f$ has a decomposition $f=\varphi \circ h$, where $h$ is a self-homeomorphism of $\mathbf{C}$ and $\varphi$ is a complex polynomial. 
If $f$ is a discrete complex polynomial for $\mathbb{H}_{n}$ and $\mathcal{B}$ is as in (i), then $\mathcal{B}$ is called the range packing of $f$.

The following theorem is a result of Corollary 4.9 and Lemma 5.2 from [D2].

\section{Theorem 2.6.}

(1) If $\mathfrak{B}=\left\{b_{1}, \ldots, b_{m}\right\}$ is a branch structure for $\mathbb{H}_{n}, n \geq 1$, then there exists a circle packing $\mathcal{B}$ for $\mathbb{H}_{n}$ with the branch set $\mathfrak{B}$ such that the cp-map $f: \mathbf{C} \rightarrow \mathbf{C}$ from $\mathcal{P}_{n}$ to $\mathcal{B}$ is a discrete complex polynomial.

(2) There exists a constant $\kappa \geq 1$, depending only on $m$, such that if $\mathcal{B}$ is a circle packing for $\mathbb{H}_{n}, n \geq 1$, and the cp-map from $\mathcal{P}_{n}$ to $\mathcal{B}$ has valence at most $m+1$, then $r_{\mathcal{B}}(w) / r_{\mathcal{B}}(v) \in(1 / \kappa, \kappa)$ for any neighboring vertices $w, v$, where $r_{\mathcal{B}}$ is the radius function of $\mathcal{B}$. In particular, any discrete complex polynomial for $\mathbb{H}_{n}, n \geq 1$, with the branch set containing at most $m$ points is $K$-quasi-regular, $K=K(m)$.

The above theorem is a key factor in a proof of an approximation result for discrete complex polynomials which will be stated shortly. However, first we need to lay down some foundations. Let $F$ be a classical complex polynomial with the critical points $x_{1}, \ldots, x_{m}$ of orders $k_{1}, \ldots, k_{m}$, respectively, i.e., $\mathfrak{B}=\left\{\left(x_{1}, k_{1}\right), \ldots,\left(x_{m}, k_{m}\right)\right\}$ is the branch set of $F$. For each sufficiently large $n$ let

$$
\mathfrak{B}_{n}=\left\{b_{1,1}(n), \ldots, b_{1, k_{1}}(n), \ldots, b_{m, 1}(n), \ldots, b_{m, k_{m}}(n)\right\}
$$

be a branch structure for $\mathbb{H}_{n}$. Further, suppose the sequence $\left\{\mathfrak{B}_{n}\right\}$ has the property that $\lim _{n \rightarrow \infty} b_{i, j}(n)=x_{i}$ for $j=1, \ldots, k_{i}$ and each $i, 1 \leq i \leq m$. It is easy to see that such a sequence $\left\{\mathfrak{B}_{n}\right\}$ can be constructed for all sufficiently large $n$. As a consequence of Theorem 5.3 and Remark 5.4 of [D2] we have

Theorem 2.7. For each sufficiently large $n$ there exists a discrete complex polynomial $f_{n}$ for $\mathbb{H}_{n}$ with the branch set $\mathfrak{B}_{n}$ such that the functions $f_{n}$ and $f_{n}^{\#}$ converge uniformly on compacta of $\mathbf{C}$ to $F$ and $\left|F^{\prime}\right|$, respectively.

The uniform convergence of $f_{n}^{\#} \rightarrow\left|F^{\prime}\right|$ in the last theorem follows from the following result that we will need later (Theorem 1 of [DS]).

Theorem 2.8. Let $\Omega \subset \mathrm{C}$ be a bounded simply connected domain. Let $\left\{\mathcal{Q}_{n}\right\}$ and $\left\{\widetilde{\mathcal{Q}}_{n}\right\}$ be sequences of circle packings such that for each $n$ the packing $\mathcal{Q}_{n}$ is univalent, $\operatorname{carr}\left(\mathcal{Q}_{n}\right) \subseteq \Omega$, and $\mathcal{Q}_{n}$ and $\widetilde{\mathcal{Q}}_{n}$ are c-equivalent. Moreover, suppose that the sets carr $\left(\mathcal{Q}_{n}\right)$ exhaust $\Omega$ and that the supremum of radii of circles in $\mathcal{Q}_{n}$ goes to 0 uniformly on compacta of $\Omega$ as $n \rightarrow \infty$. In addition, assume that the functions $g_{n}$ converge uniformly on compacta of $\Omega$ to an analytic function $g: \Omega \rightarrow \mathrm{C}$ as $n \rightarrow \infty$, where $g_{n}: \operatorname{carr}\left(\mathcal{Q}_{n}\right) \rightarrow \operatorname{carr}\left(\widetilde{\mathcal{Q}}_{n}\right)$ is the cp-map from $\mathcal{Q}_{n}$ to $\widetilde{\mathcal{Q}}_{n}$. Then the ratio functions $g_{n}^{\#}$ converge uniformly on compacta of $\Omega$ to $\left|g^{\prime}\right|$. 


\section{The Main Result}

In this section we are concerned with the following problem. Let $\Omega \subset \mathbf{C}$ be a Jordan domain. Let $0 \in \Omega$ and $\xi \in \Omega, \xi>0$. Suppose $\lambda: \partial \Omega \rightarrow(0, \infty)$ is a continuous function and $\mathfrak{B}=\left\{\left(x_{1}, k_{1}\right), \ldots,\left(x_{m}, k_{m}\right)\right\}$ is a subset of $\Omega \times \mathbf{N}$. It is a well-known fact that there exists the unique analytic function $F$ in $\Omega$ such that $F(0)=0, F(\xi)>0$, the set of critical points of $F$ is equal to $\mathfrak{B}$, and $\left|F^{\prime}\right|$ has a continuous extension to $\bar{\Omega}$ with $\left|F^{\prime}\right| \equiv \lambda$ on $\partial \Omega$. We are interested in the approximation of $F$ using cp-maps having only been given $\Omega, \lambda, \mathfrak{B}$, and $\xi$.

We show that this can be achieved, roughly speaking, by taking the cp-map from a portion of a regular hexagonal circle packing of suitable small mesh that fills up $\Omega$ to a c-equivalent circle packing whose branch set approximates $\mathfrak{B}$ and whose radius function on the boundary is approximately equal to $\lambda$ times the mesh of the hexagonal packing. The precise setup is as follows.

Write $\mathbb{O}_{n}$ for the maximal complete subcomplex of $\mathbb{H}_{n}$ contained in $\Omega$, that is, a simply connected simplicial 2-complex consisting of all faces of $\mathbb{H}_{n}$ whose closures are in $\Omega$, together with their edges and vertices. Denote $\mathcal{Q}_{n}$ to be the portion of $\mathcal{P}_{n}$ associated with $\mathbb{O}_{n}$, where $\mathcal{P}_{n}$ is as in Section 2 . Suppose that for each sufficiently large $n$ there exists a circle packing $\widetilde{\mathcal{Q}}_{n}$ c-equivalent to $\mathcal{Q}_{n}$ such that

(1) if $r_{n}: \mathbb{O}_{n}^{(0)} \rightarrow(0, \infty)$ is the radius function of $\widetilde{\mathcal{Q}}_{n}$ and $v \in$ bd $\mathbb{O}_{n}^{(0)}$, then $r_{n}(v)=$ $(1 / n) \lambda\left(z_{v}\right)$, where $z_{v}$ is a point on $\partial \Omega$ closest to $v$,

(2) if the set $\mathfrak{B}_{n}=\left\{b_{1,1}(n), \ldots, b_{1, k_{1}}(n), \ldots, b_{m, 1}(n), \ldots, b_{m, k_{m}}(n)\right\}$ of distinct vertices of $\mathbb{O}_{n}$ is the branch set of $\mathcal{Q}_{n}$, then the sequence $\left\{\mathfrak{B}_{n}\right\}$ has the property that $\lim _{n \rightarrow \infty} b_{i, j}(n)=x_{i}$ for $j=1, \ldots, k_{i}$ and each $i, 1 \leq i \leq m$,

(3) if $f_{n}: \operatorname{carr}\left(\mathcal{Q}_{n}\right) \rightarrow \operatorname{carr}\left(\widetilde{\mathcal{Q}}_{n}\right)$ is the cp-map from $\mathcal{Q}_{n}$ to $\widetilde{\mathcal{Q}}_{n}$, then $f_{n}(0)=0$ and $f_{n}(\xi)>0$,

then $\left\{\widetilde{\mathcal{Q}}_{n}\right\}$ is called an approximating sequence of circle packings for $F$. We prove the following theorem which is our main result.

Theorem 3.1. Let $\Omega \subset \mathbf{C}$ be a Jordan domain. Let $0 \in \Omega$ and $\xi \in \Omega, \xi>0$. Suppose $\lambda: \partial \Omega \rightarrow(0, \infty)$ is a continuous function and $\mathfrak{B}=\left\{\left(x_{1}, k_{1}\right), \ldots,\left(x_{m}, k_{m}\right)\right\}$ is a subset of $\Omega \times \mathbf{N}$. Let $F$ be the unique analytic function in $\Omega$ such that $F(0)=0, F(\xi)>0$, the set of critical points of $F$ is equal to $\mathfrak{B}$, and $\left|F^{\prime}\right|: \bar{\Omega} \rightarrow[0, \infty)$ is continuous and $\left|F^{\prime}\right|_{\partial \Omega} \equiv \lambda$. Then there exists an approximating sequence $\left\{\widetilde{\mathcal{Q}}_{n}\right\}$ of circle packings for $F$. Moreover, if the maps $f_{n}$ are defined as in (3) above, then $f_{n}$ and $f_{n}^{\#}$ converge uniformly on compacta of $\Omega$ to $F$ and $\left|F^{\prime}\right|$, respectively.

Before we give a proof of the above theorem we make two remarks.

Remark 3.2. We observe that our construction of an approximating sequence $\left\{\widetilde{\mathcal{Q}}_{n}\right\}$ is based exclusively on $\Omega, \lambda, \mathfrak{B}$, and $\xi$, hence it gives a constructive method for the approximation of $F$.

Remark 3.3. If $\mathfrak{B}=\emptyset$, then we obtain the results of [CR] and [CVM]. 

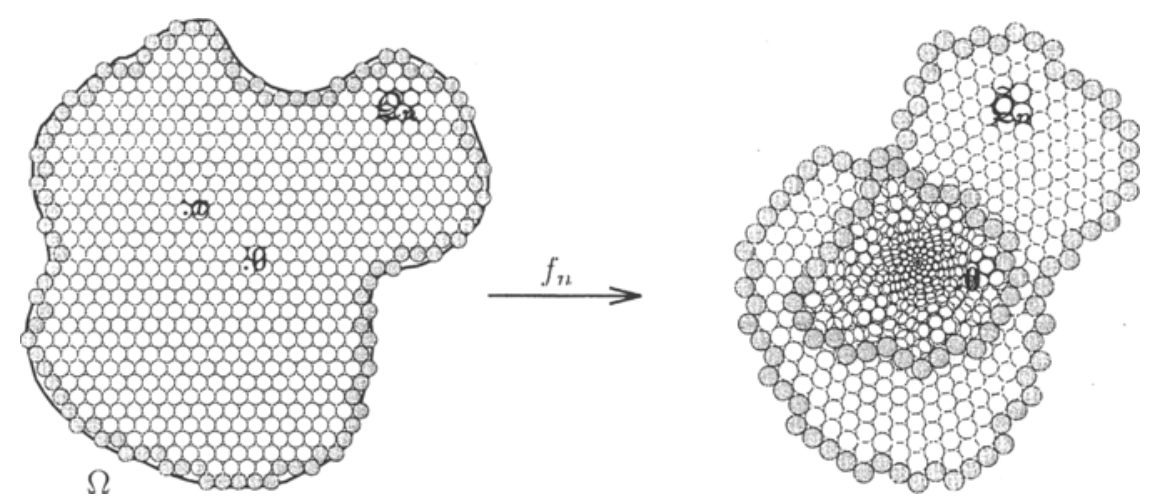

Fig. 2. The circle-packing map in an approximation sequence: $\lambda \equiv 1.4, \mathfrak{B}=\{(x, 1)\}$.

To prove Theorem 3.1 we need the following:

Lemma 3.4. Let $\Omega$ and $F$ be as in Theorem 3.1. Then there exists a sequence of polynomials $\left\{\chi_{n}\right\}$ such that $\chi_{n} \rightarrow F$ and $\left|\chi_{n}^{\prime}\right| \rightarrow\left|F^{\prime}\right|$ uniformly in $\bar{\Omega}$ as $n \rightarrow \infty$.

Proof. First we need a formula for $F$. Let $u$ be the harmonic function in $\Omega$ with $\left.u\right|_{\partial \Omega}=\log \lambda$. Denote by $v$ the harmonic conjugate of $u$ in $\Omega$. Write $\tau: \Omega \rightarrow D$ for the Riemann mapping with $\tau(0)=0$ and $\tau(\xi)>0$. Then the function $F$ is given by

$$
F(z)=\int_{0}^{z} B(\tau(\eta)) e^{u(\eta)+i v(\eta)} d \eta
$$

where

$$
B(\zeta)=c \prod_{i=1}^{m}\left(\frac{\zeta-\tau\left(x_{i}\right)}{1-\overline{\tau\left(x_{i}\right) \zeta}}\right)^{k_{i}}
$$

and $c$ is a suitable unimodular constant (such that $F(\xi)>0$ ).

Let $\left\{\Omega_{n}\right\}$ be a sequence of Jordan domains such that $\bar{\Omega} \subset \Omega_{n+1} \subset \Omega_{n}, n \geq 1$, and the boundary of $\Omega_{n}$ converges to $\partial \Omega$ in the sense of Fréchet (see [LV, p. 27], [H], and [W]). Write $\tau_{n}: \Omega_{n} \rightarrow D$ for the Riemann mapping with $\tau_{n}(0)=0$ and $\tau_{n}(\xi)>0$. Then $\tau_{n} \rightarrow \tau$ uniformly in $\bar{\Omega}$. Define $F_{n}: \Omega_{n} \rightarrow \mathrm{C}$ by

$$
F_{n}(z)=\int_{0}^{z} B\left(\tau_{n}(\eta)\right) e^{u\left(\tau^{-1} \circ \tau_{n}(\eta)\right)+i v\left(\tau^{-1} \circ \tau_{n}(\eta)\right)} d \eta
$$

Then $F_{n} \rightarrow F$ uniformly in $\bar{\Omega}$. Moreover, as $\left|F_{n}^{\prime}(z)\right|=\left|B\left(\tau_{n}(z)\right)\right| e^{\mu\left(\tau^{-1} \circ \tau_{n}(\eta)\right)}$ for $z \in$ $\bar{\Omega}$, and $B$ and $u$ are continuous in $\bar{D}$ and $\bar{\Omega}$, respectively, we also get $\left|F_{n}^{\prime}\right| \rightarrow\left|F^{\prime}\right|$ uniformly in $\bar{\Omega}$. By Runge's theorem for each $n$ there exists a polynomial $\chi_{n}$ such that $\sup _{z \in \bar{\Omega}}\left|F_{n}(z)-\chi_{n}(z)\right|<1 / n$ and $\sup _{z \in \bar{\Omega}}\left|F_{n}^{\prime}(z)-\chi_{n}^{\prime}(z)\right|<1 / n$. The last implies the assertion of the lemma.

We are now ready to prove Theorem 3.1. 
Proof of Theorem 3.1. We first need to verify the existence of an approximating sequence of circle packings for the function $F$. This follows easily from Theorem 2.3, Definition 2.1, and the geometry of simplicial complexes $\mathbb{O}_{n}$. In fact, Definition 2.1 and the geometry of simplicial complexes $\mathbb{O}_{n}$ are only needed to obtain condition (2) in the definition of an approximating sequence. Condition (3) of that definition is achieved immediately by applying translations and/or rotations, if required, to packings already constructed.

We now show that if $\left\{\widetilde{\mathcal{Q}}_{n}\right\}$ is an approximating sequence of circle packings for $F$, then the maps $f_{n}: \operatorname{carr}\left(\mathcal{Q}_{n}\right) \rightarrow \operatorname{carr}\left(\widetilde{\mathcal{Q}}_{n}\right)$ and $f_{n}^{\#}: \operatorname{carr}\left(\mathcal{Q}_{n}\right) \rightarrow(0, \infty)$ converge uniformly on compacta of $\Omega$ to $F$ and $\left|F^{\prime}\right|$, respectively. Let $G$ be a complex polynomial with the branch set $\mathfrak{B}$ such that $G(0)=0$ and $G(\xi)=1$. If $\mathfrak{B}_{n}$ is the branch set of $\widetilde{\mathcal{Q}}_{n}$, then it is not hard to see that $\mathfrak{B}_{n} \subset \mathbb{O}_{n}^{(0)} \subset \mathbb{H}_{n}^{(0)}$ is a branch structure for $\mathbb{H}_{n}$ for all sufficiently large $n$. From Theorem 2.7 there exists, for each sufficiently large $n$, a discrete complex polynomial $g_{n}$ for $\mathbb{H}_{n}$ with the branch set $\mathfrak{B}_{n}$ such that $g_{n} \rightarrow G$ and $g_{n}^{\#} \rightarrow\left|G^{\prime}\right|$ uniformly on compacta of $\mathbf{C}$ as $n \rightarrow \infty$. Write $R_{n}: \mathbb{H}_{n}^{(0)} \rightarrow(0, \infty)$ for the radius function of the range packing $\mathcal{U}_{n}$ of $g_{n}$. Since $\left.\left|G^{\prime}\right|\right|_{\partial \Omega}>0,\left.\left.g_{n}^{\#}\right|_{\partial \Theta_{n}} \underset{n \rightarrow \infty}{\longrightarrow}\left|G^{\prime}\right|\right|_{\partial \Omega}$, and $g_{n}^{\#}(v)=R_{n}(v) /(1 / n)$, there exists $\sigma \geq 1$ such that $1 / n \sigma \leq\left. R_{n}\right|_{\mathrm{bd} O_{n}^{(0)}} \leq \sigma / n$ for all $n$. Denote by $\tilde{U}_{n}$ the portion of $\mathcal{U}_{n}$ associated with the subcomplex $\mathbb{O}_{n}$ of $\mathbb{H}_{n}$. Recall that $r_{n}: \mathbb{O}_{n}^{(0)} \rightarrow(0, \infty)$ is the radius function of $\widetilde{\mathcal{Q}}_{n}$. Since $\widetilde{\mathcal{U}}_{n}$ and $\widetilde{\mathcal{Q}}_{n}$ are packings for $\mathbb{O}_{n}$ with the branch sets $\mathfrak{B}_{n}$, Fact 2.4 implies that $\left(r_{n} / R_{n}\right)(\cdot): \mathbb{O}_{n}^{(0)} \rightarrow(0, \infty)$ attains its maximum and minimum in bd $\mathbb{O}_{n}^{(0)}$. Hence

$$
\frac{1}{\sigma} \min _{\partial \Omega} \lambda \leq \frac{r_{n}}{\left.R_{n}\right|_{\mathbb{O}_{n}^{(0)}}} \leq \sigma \max _{\partial \Omega} \lambda
$$

because $(1 / \sigma) \min _{\partial \Omega} \lambda \leq\left.\left(r_{n} / R_{n}\right)\right|_{\mathrm{bd} \mathrm{O}_{n}^{(0)}} \leq \sigma \max _{\partial \Omega} \lambda$. Now it follows from Theorem 2.6 (applied to $g_{n}$ ) and (3.1) that there exists a constant $\kappa \geq 1$ such that

$$
\frac{1}{\kappa} \leq \frac{r_{n}(w)}{r_{n}(v)} \leq \kappa \quad \text { for any } n \text { and any neighboring vertices } v, w \in \mathbb{O}_{n}^{(0)} .
$$

The last conclusion and the construction of the maps $f_{n}$ imply that $\left\{f_{n}\right\}$ is a family of $K$ quasi-regular mappings for some $K \geq 1$. Moreover, since $\Omega$ is bounded and by Fact 2.4 the ratio functions $f_{n}^{\#}$ are uniformly bounded by $\max _{\partial \Omega} \lambda$, it follows that the maps $f_{n}$ are uniformly bounded. Hence $\left\{f_{n}\right\}$ is a normal family. By taking a subsequence if necessary, assume that $f_{n} \rightarrow f$ uniformly on compacta of $\Omega$ as $n \rightarrow \infty$. We first show that the limit function $f$ is not constant. If $f$ were constant then, by Theorem 2.8 , functions $f_{n}^{\#}$ would converge uniformly on compacta of $\Omega$ to the constant zero-function. However, this would contradict (3.1) for sufficiently large $n$ because $\left.\left(r_{n} / R_{n}\right)\right|_{\mathbb{O}_{n}^{(0)}}=\left.l\left(f_{n}^{\#} / g_{n}^{\#}\right)\right|_{\mathcal{O}_{n}^{(0)}}$ and $\left|G^{\prime}\right|>0$ in $\Omega \backslash\left\{x_{1}, \ldots, x_{m}\right\}$. Hence $f$ is nonconstant $K$-quasi-regular mapping in $\Omega$.

We now verify that $f$ is actually analytic. Since $f_{n}$ is bounded $K$-quasi-regular mapping, by Stoilow's theorem [LV], it has a decomposition $f_{n}=\varphi_{n} \circ \psi_{n}$, where $\psi_{n}: \mathbb{O}_{n} \rightarrow D$ is a K-quasi-conformal homeomorphism normalized by $\psi_{n}(0)=0$ and $\psi_{n}(\xi)>0$, and $\varphi_{n}: D \rightarrow \mathbf{C}$ is an analytic function with $\varphi_{n}(0)=0$ and $\varphi_{n}\left(\psi_{n}(\xi)\right)>0$. By extracting a subsequence from $\left\{f_{n}\right\}$ if necessary, we can assume that $\varphi_{n} \rightarrow \varphi$ and $\psi_{n} \rightarrow \psi$ uniformly on compacta of $\Omega$ and $D$, respectively, where $\psi: \Omega \rightarrow D$ is a Kquasi-conformal homeomorphism, $\varphi: D \rightarrow \mathrm{C}$ is analytic, and $f=\varphi \circ \psi$. In particular, $\varphi$ 
and $\psi$ are not constant and the branch set of $\varphi$ is equal to $\left\{\left(\psi\left(x_{1}\right), k_{1}\right), \ldots,\left(\psi\left(x_{m}\right), k_{m}\right)\right\}$. Let $z_{0} \in \Omega \backslash\left\{x_{1}, \ldots, x_{m}\right\}$. From the equicontinuity of the normal family $\left\{\psi_{n}\right\}$ and its uniform convergence on compacta to the function $\psi$ we get that there exist $\varepsilon>0$ and $n_{0}$ such that $\left.f_{n}\right|_{B\left(z_{0}, \varepsilon\right)}$ is one-to-one for $n>n_{0}$, where $B\left(z_{0}, \varepsilon\right)=\left\{\left|z-z_{0}\right|<\varepsilon\right\}$. Let $\widetilde{\mathcal{Q}}_{n}\left(z_{0}\right)$ be the portion of $\widetilde{\mathcal{Q}}_{n}$ associated with the largest complete hexagonal generation of $\mathbb{O}_{n}$ around $z_{0}$ contained in $B\left(z_{0}, \varepsilon\right)$. Then $\left\{\widetilde{\mathcal{Q}}_{n}\left(z_{0}\right)\right\}$ is a sequence of univalent hexagonal circle packings with their number of generations around $z_{0}$ going to $\infty$ as $n \rightarrow \infty$. From the Hexagonal Packing Lemma of [RS] (see also [H], [M], and [Sc]) we conclude that the quasi-conformal distortion of the maps $f_{n}$ at $z_{0}$ goes to 0 as $n \rightarrow \infty$. Hence the quasi-conformal distortion of the mappings $\psi_{n}$ at $z_{0}$ goes to 0 as $n \rightarrow \infty$. Thus $\psi$ is 1-quasi-conformal in $\Omega \backslash\left\{x_{1}, \ldots, x_{m}\right\}$. Since $\psi$ is a homeomorphism of $\Omega$, the last implies that $\psi$ is conformal in $\Omega$ (see [LV, Chapter I, Theorem 8.1] and [A, Chapter II, Corollary 2]). Therefore $f=\varphi \circ \psi$ is analytic in $\Omega$ with the branch set $\mathfrak{B}$.

To complete our proof we need to show that $f=F$. To achieve this we have to prove that $\left|f^{\prime}\right|$ has a continuous extension to $\partial \Omega$ and is equal to $\lambda$ there. We observe first that since $f$ is analytic in $\Omega$, by Theorem $2.8, f_{n}^{\#} \rightarrow\left|f^{\prime}\right|$ uniformly on compacta of $\Omega$ as $n \rightarrow \infty$. Let $\left\{\chi_{n}\right\}$ be a sequence of polynomials given by Lemma 3.4. Since $\left.\left|F^{\prime}\right|\right|_{\partial \Omega}>0$ and $F$ has a finite branch set in $\Omega$, we can assume that for each $n$ the restriction of the branch set of $\chi_{n}$ to $\bar{\Omega}$ is equal to the branch set $\mathfrak{B}$ of $F$. Take $\delta>0$ such that $\left|F^{\prime}(z)\right|>0$ for $z \in \Omega^{\delta}:=\{z \in \bar{\Omega}$ : $\operatorname{dist}(z, \partial \Omega) \leq \delta\}$. Given $\varepsilon>0$ let $N(\varepsilon)$ be such that

$$
|| \chi_{n}^{\prime}(z)|-| F^{\prime}(z)||<\varepsilon, \quad z \in \bar{\Omega}, \quad n \geq N(\varepsilon)
$$

and

$$
\left|\frac{\left|\chi_{n}^{\prime}(z)\right|}{\left|F^{\prime}(z)\right|}-1\right|<\varepsilon, \quad z \in \Omega^{\delta}, \quad n \geq N(\varepsilon) .
$$

From the geometry of the complexes $\mathbb{H}_{n}$, Theorem 2.7 , and the fact that $\mathfrak{B}_{n}$ is a branch structure for $\mathbb{O}_{n}$, it follows that there exists a sequence of discrete polynomials $\left\{p_{n}\right\}$ such that $p_{n}$ is a discrete polynomial for $\mathbb{H}_{n}, \operatorname{br}\left(p_{n}\right) \cap \bar{\Omega}=\mathfrak{B}_{n}$, and $p_{n} \rightarrow \chi_{N(\varepsilon)}$ and $p_{n}^{\#} \rightarrow\left|\chi_{N(\varepsilon)}^{\prime}\right|$ uniformly on compacta of $\mathbf{C}$ as $n \rightarrow \infty$. Now (3.4) implies that there is $N^{\prime}(\varepsilon), N^{\prime}(\varepsilon) \geq N(\varepsilon)$, such that

$$
\left|\frac{p_{n}^{\prime \prime}(z)}{\left|F^{\prime}(z)\right|}-1\right|<2 \varepsilon, \quad z \in \Omega^{\delta}, \quad n \geq N^{\prime}(\varepsilon) .
$$

Write $\mathcal{W}_{n}$ for the image packing of $p_{n}$. Denote by $\widetilde{\mathcal{W}}_{n}$ the portion of $\mathcal{W}_{n}$ associated with the subcomplex $\mathbb{O}_{n}$ of $\mathbb{H}_{n}$, and by $\varrho_{n}$ the radius function of $\mathcal{W}_{n}$. Since $\widetilde{\mathcal{W}}_{n}$ and $\widetilde{\mathcal{Q}}_{n}$ are circle packings for $\mathbb{O}_{n}$ with identical branch sets, Fact 2.4 shows that $\left(\varrho_{n} / r_{n}\right)(\cdot): \mathbb{O}_{n}^{(0)} \rightarrow(0, \infty)$ has its maximum and minimum in bd $\mathbb{O}_{n}^{(0)}$. Hence $\left.\left(p_{n}^{\#} / f_{n}^{\#}\right)\right|_{\mathbb{O}_{n}^{(0)}}: \mathbb{O}_{n}^{(0)} \rightarrow(0, \infty)$ has its maximum and minimum in bd $\mathbb{O}_{n}^{(0)}$. From the last conclusion, the construction of $\widetilde{\mathcal{Q}}_{n}$ (i.e., $\left.\left.f_{n}^{\#}\right|_{\partial\left(\operatorname{carr}\left(\mathcal{Q}_{n}\right)\right)} \approx\left|F^{\prime}\right|_{\partial\left(\operatorname{carr}\left(\mathcal{Q}_{n}\right)\right)} \approx \lambda\right)$, the fact that $\partial \operatorname{carr}\left(\mathcal{Q}_{n}\right) \subset \Omega^{\delta}$ for large $n$, and (3.5), it follows that there exists $N^{\prime \prime}(\varepsilon), N^{\prime \prime}(\varepsilon) \geq N^{\prime}(\varepsilon)$, such that

$$
\left|\frac{p_{n}^{\#}}{f_{n}^{\#}}(v)-1\right|<3 \varepsilon, \quad v \in \mathbb{O}_{n}^{(0)}, \quad n \geq N^{\prime \prime}(\varepsilon) .
$$


We observe that $\left.f_{n}^{\#}\right|_{\mathbb{O}_{n}^{(0)}} \leq \max _{\partial \Omega} \lambda$ by Fact $2.4(2)$. Hence, since $p_{n}^{*}$ and $f_{n}^{\#}$ have been defined as simplicial extensions of $\left.p_{n}^{\#}\right|_{\mathbb{O}_{n}^{(0)}}$ and $\left.f_{n}^{\#}\right|_{\mathbb{O}_{n}^{(0)}}$, respectively, we obtain from (3.6)

$$
\left|p_{n}^{\#}(z)-f_{n}^{\#}(z)\right|<3 \varepsilon \max _{\partial \Omega} \lambda, \quad z \in \operatorname{carr}\left(\mathcal{Q}_{n}\right), \quad n \geq N^{\prime \prime}(\varepsilon) .
$$

Recall that $f_{n}^{\#} \rightarrow\left|f^{\prime}\right|$ and $p_{n}^{\#} \rightarrow\left|\chi_{N(\varepsilon)}^{\prime}\right|$ uniformly on compacta of $\Omega$. Thus, by letting $n \rightarrow \infty$ in (3.7), we get

$$
|| \chi_{N(\varepsilon)}^{\prime}|-| f^{\prime}(z)||<3 \varepsilon \max _{\partial \Omega} \lambda, \quad z \in \Omega .
$$

As $\varepsilon$ was arbitrary, (3.4) implies that $\left|F^{\prime}\right| \equiv\left|f^{\prime}\right|$ in $\Omega$. In particular, $\left|f^{\prime}\right|$ has a continuous extension to $\partial \Omega$ and is equal to $\left|F^{\prime}\right|_{\partial \Omega} \equiv \lambda$ there. Hence $f \equiv F$ and the proof is complete.

\section{Concluding Remarks}

(1) Notice that Theorem 3.1 gives, in essence, a method for the approximation of the integral of an analytic function on compacta of its domain.

(2) Since the sequence of maps $\left\{f_{n}^{\#}\right\}$ converges uniformly on compacta of $\Omega$ to $\left|F^{\prime}\right|$, $\left\{\log f_{n}^{\#}\right\}$ converges uniformly on compacta of $\Omega \backslash\left\{x_{1}, \ldots, x_{m}\right\}$ to the harmonic function $u$ in $\Omega \backslash\left\{x_{1}, \ldots, x_{m}\right\}^{\prime}$ which has isolated singularities at $x_{i}$ of degree $k_{i}, i=1, \ldots, m$, and which satisfies boundary condition $\left.u\right|_{\partial \Omega}=\log \lambda$. We also note that the functions $f_{n}^{\#}$ are easier to construct than the functions $f_{n}$; the latter require the radius functions of $\widetilde{\mathcal{Q}}_{n}$ 's and the centers of circles in $\widetilde{\mathcal{Q}}_{n}$ 's, while the former require only radius functions of $\overline{\mathcal{Q}}_{n}$ 's. Moreover, the reader might be interested to learn that some estimates on the speed of convergence of $f_{n}^{\#} \rightarrow\left|F^{\prime}\right|$ were established in [CVM].

(3) Arguments presented so far in this paper can easily be extended to other combinatorial patterns as follows. Let $\mathcal{O}$ be a univalent circle packing whose carrier is $\mathbf{C}$ (e.g., the "ball-bearing" packing - see Fig. 2(b) of [BS]). Denote the geometric complex of $\mathcal{O}$ by $\mathbb{L}$. Assume that $\mathbb{L}$ is of bounded degree, i.e., there is a uniform bound on the number of neighboring vertices of each vertex in $\mathbb{L}$. Further, suppose $\mathcal{O}$ has the property that for each $\varepsilon>0$ there is $n_{\varepsilon}$ such that all circles of $(1 / n) \mathcal{O}, n \geq n_{\varepsilon}$, contained in $\{|z|<1\}$ have their radii at most $\varepsilon$. Then Definition 2.5 and Theorem 2.6 can be generalized to discrete complex polynomials for $\mathbb{L}_{n}(=(1 / n) \mathbb{L})$ as was done in [D2]. Moreover, Theorem 2.7 for the complexes $\mathbb{L}_{n}$ can be obtained by following the steps of the proof of Theorem 5.3 in [D2] and using Theorem 2.2 of [HR]. It is now a matter of replacing $\mathbb{H}_{n}$ by $\mathbb{L}_{n}$ to define approximation sequences based on complex $\mathbb{L}$ and to verify Theorem 3.1 for such sequences.

\section{References}

[A] L. Ahlfors, Lectures on Quasiconformal Mappings, Wadsworth \& Brooks/Cole, Monterey, CA, 1987.

[BS] A. F. Beardon and K. Stephenson, The uniformization theorem for circle packings, Indiana Univ. Math. J. 39 (1990), 1383-1425.

[CR] I. Carter and B. Rodin, An inverse problem for circle packing and conformal mapping, Trans. Amer. Math. Soc. 334 (1992), 861-875. 
[CVM] Y. Colin de Verdière and F. Mathéus, Empilements de cercles et approximation conformes, info à paraître dans les Actes de la Table Ronde de Géométrie Riemannienne en l'honneur de Marcel Berger, Arthur Besse (Editeur), collection SMF, Séminaires et congrés 1 (1995).

[D1] T. Dubejko, Branched circle packings and discrete Blaschke products, Trans. Amer. Math. Soc. 347(95), 4073-4103.

[D2] T. Dubejko, Infinite branched circle packings and discrete complex polynomials J. London Math. Soc. (to appear).

[DS] T. Dubejko and K. Stephenson, The branched Schwarz lemma: a classical result via circle packing, Michigan Math. J. 42 (1995), 21 1-234.

[G] B. T. Garrett, Circle packings and polyhedral surfaces, Discrete Comput. Geom. 8 (1992), 429-440.

[H] Z.-X. He, An estimate for hexagonal circle packings, J. Differential Geom. 33 (1991), 395-412.

[HR] Z.-X. He and B. Rodin, Convergence of circle packings of finite valence to Riemann mappings, Comm. Anal. Geom. 1 (1993), 31-41.

[LV] O. Lehto and K. I. Virtanen, Quasiconformal Mappings in the Plane, 2nd edn., Springer-Verlag, New York, 1973

[M] F. Mathéus, Empilements de cercles: rigidité, discrétisation d'application conformes, Thése de Doctorat, Université Grenoble 1, 1994.

[RS] B. Rodin and D. Sullivan, The convergence of circle packings to the Riemann mapping, J. Differential Geom. 26 (1987), 349-360.

[Sc] O. Schramm, Rigidity of infinite (circle) packings, J. Amer. Math. Soc. 4 (1991), 127-149.

[St] K. Stephenson Circle packings in the approximation of conformal mappings, Bull. Amer. Math. Soc. 23(2) (1990), 407-415.

[W] S. E. Warschawski, On the degree of variation in conformal mapping of variable regions, Trans. Amer. Math. Soc. 69 (1950), 335-356.

Received January 25, 1995, and in revised form December 11, 1995. 